\title{
An Approach to Representing Movement Data
}

\author{
Hong Thi Nguyen, Hung Thanh Ngo, Xanh Van Nguyen, Diem Ngoc Nguyen, and Phuoc Vinh Tran, \\ Senior Member, IACSIT
}

\begin{abstract}
A moving object is an object of which position changes continuously in space. The movement of an object is represented by the change of its position through space and the change of its attributes over time or location. In temporal geography, the model of space-time cube (STC) represents movements as polylines time-ordered connecting spatio-temporal positions of moving objects. The space-time cubes do not represent attributes of moving objects, which change over time or along trajectories. Several authors used two cubes to represent a moving object, one for spatio-temporal positions and another for attributes changing over location. This article proposes an approach to representing multivariate data of moving objects in a Cartesian coordinate system. The main idea of this article is to join the coordinate system representing location data with the coordinate system representing attributes by sharing the time axis. In this case, attributes that are recorded by the location-based method are inferred visually through the space-time path of the movement. This approach can apply for two cases, given route and ungiven route. For movements of ungiven route, the visualization system combines a 3-dimension system representing locations with a 2-dimension system representing attributes. For movements of given route, the visualization system is a 3-dimension Cartesian coordinate system, where the trajectory is extended as an axis representing the distance from departure place to current place, two other axes indicate time and attributes. In both modes, colors are employed as dimensions to show various attributes. The two modes can be switched reciprocally in a system tracking moving objects on ground.
\end{abstract}

Index Terms-Data visualization, spatio-temporal representation, movement data, space-time cube, multivariate data.

\section{INTRODUCTION}

A map is a geographic representation to indicate positions in space of entities, phenomena, and events, which are called jointly objects. In traditional 2-dimension maps, the movements of objects are represented as route maps to indicate the trajectories of the objects. Another approach of movement map is to integrate flow charts into 2-dimension maps to show not only the trajectories of moving objects, but also their attributes. In temporal geography, the model of space-time cube uses 3-dimension Cartesian coordinate systems to indicate positions of objects in space and in time

Manuscript received November 3, 2012; revised December 6, 2012. This work was supported in part by a budget from the Advanced Program of University of Information Technology (UIT), granted by Vietnam's Ministry of Education and Training.

Hong Thi Nguyen, Hung Thanh Ngo, Xanh Van Nguyen, Diem Ngoc Nguyen, and Phuoc Vinh Tran are with the University of Information Technology (UIT), Hochiminh City, Vietnam (e-mail: hongnguyen1611@gmail.com, hungnt@uit.edu.vn, xanhnv@uit.edu.vn, ngngdiem@gmail.com, phuoc.gis@uit.edu.vn,phuoc.gis@gmail.com).
[1], [2], [3], [4]. A space-time cube does not indicate object attributes varying over time or location [5]. In this paper, we propose a model of Multivariate Cube (MC) to represent moving objects, of which spatial positions and attributes change over time.

The main idea of this article is to combine two Cartesian coordinate systems representing data, a system represents the positions changing over time and a system represents attributes changing over time. The two systems share the time axis to join time references of both position data and attribute data. According to the approach, attributes are represented in a 2-dimension Cartesian coordinate system, of which an axis indicates time, another indicates attributes. Colors are employed as different dimensions to indicate various attributes [6]. The positions of objects are indicated in a Cartesian coordinate system of 2 dimensions or 3 dimensions. The 2-dimension coordinate system including an axis of time and an axis of location is applied for the case of given routes to represent spatio-temporal positions of moving objects, where spatial positions are indicated on the location axis by the distance from the departure position to a study position. The 3-dimension coordinate system including an axis of time and two axes of locations is applied for the case of ungiven routes to represent spatio-temporal positions of moving objects, where spatial positions are indicated on the plane of two location axes by coordinate pairs $(x, y)$ [7], [8].

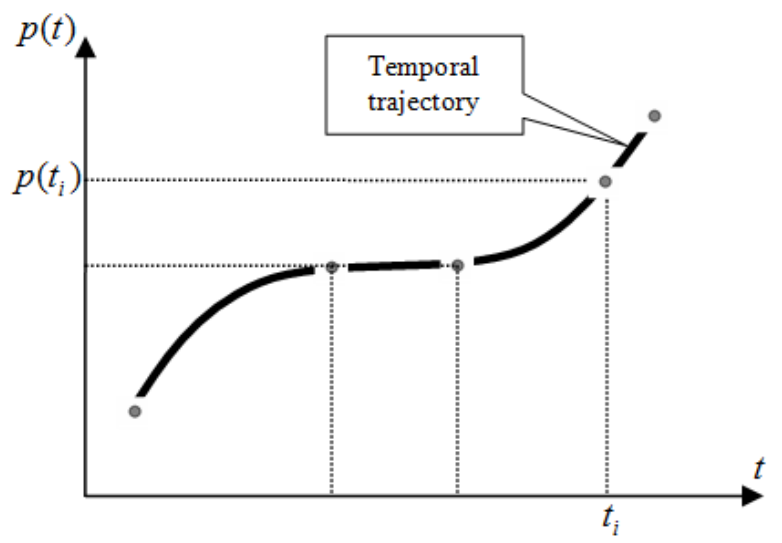

Fig. 1. A space-time path is a spatio-temporal relation

In other words, a multivariate cube represents movement data in two modes, the mode of given route and the mode of ungiven route. The coordinate system for given route mode includes a time axis, a position axis, and an attribute axis. Meanwhile, the coordinate system for ungiven route mode includes a time axis, two position axes, and an attribute axis. In reality, the two modes can be switched reciprocally in a visualization system. 
This paper is structured as follows. The next item is the summary of the concepts employed in this paper and researches concerning the representation of movement data and multivariate data. The item 3 deploys the process making data table for the visualization of moving objects. In the item 4 , the article proposes the approach of Multivariate Cube (MC) representing data of moving objects in case of given route and ungiven route. The final item is the conclusion of the article and future works.

\section{CONCEPTUAL FRAMEWORK AND RELATED WORKS}

From the triad "What, Where, When" by Peuquet in 1994, Andrienko constituted the triad "Objects, Locations, Times" and analyzed further the relations between these three sets of discrete elements [9], [10]. These relations formed the concepts of spatial objects, temporal objects, spatio-temporal objects, and moving objects. A spatial object is an object having a position determined in space at any time moments of its presence. A temporal object is an object having a position determined in time. A spatio-temporal object is an object existing at a position determined in space and at a moment determined in time [9], [11]. A moving object is an object of which spatial position changes continuously through space over time. The relation between space and time of a moving object constitutes the space-time path of the movement, which is continuous in the spatio-temporal domain (Fig.1). The continuous space-time paths of moving objects are sampled to constitute discrete spatio-temporal points corresponding to the relation between the elements of the set of locations and the elements of the set of times in the Andrienko's triad [9].

The visual representation of data refers to the display of data variables on a coordinate system. Traditionally, 2-dimension coordinate systems have been used to make geographic maps because they are suitable for the representation of geographic world. To represent moving objects, flow charts are integrated into 2-dimension maps to form flow maps showing the movement of objects from a location to another [1]. Minard's map of Napoleon's disastrous Russian campaign created in 1861 is a well-known illustration of flow maps, where Charles Joseph Minard employed flow map of a 2-dimension coordinate system to represent the data of positions, times, and the number of Napoleon's soldiers [12], [13]. Since Hagertrand included time into geographic data in 1970, he and many other authors have applied 3-dimension Cartesian coordinate systems as space-time cubes (STC) to represent data of spatio-temporal objects [14], [7], [8]. In a space-time cube, two of three axes of the coordinate system are employed to indicate spatial positions of objects, the other to indicate temporal positions.

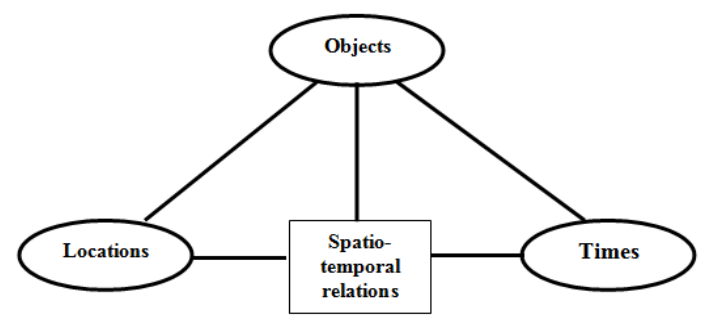

Fig. 2. The relations of a moving object

\section{To Constitute Data TABle FOR Visual REPRESENTATION OF MOVING OBJECTS}

The data table for visual representation of moving objects is constituted according to the following process:

1) Analyzing the relations between objects and locations, and times: The relations between objects, locations, and times in geographic world constitute relation tuples [15], [16]. The relation tuple <objects, locations> defines spatial objects that are objects having location determined during its presence. The relation tuple <objects, times> defines temporal objects that are objects having the time determined during its existence. The relation tuple <objects, locations, times> defines spatio-temporal objects that are objects determined positions in space and in time. The relation tuple <objects, locations, times, trajectory> defines moving objects (Fig.2). In reality, every object has several thematic attributes that have the relations with locations and/or times. In this article, we detect attributes from the set of objects to form the set of attributes (Fig. 3). The relation between the sets of attributes and locations constitutes the relation tuple <attributes, locations>, and the relation between the sets of attributes and times constitutes the relation tuple <attributes, times>.



Fig. 3. The set of Attributes is detected from the set of objects

2) Converting relation tuples into data tuples: Mathematically, the movement of an object is a function from a continuous domain of time to a continuous domain of space. In other words, a movement is a function mapping time $t$ onto position $p, p: t \mapsto p(t)$, where $t$ changes continuously and naturally, $p(t)$ is the position in space of the object. The curve $p(t)$ in spatial domain is the trajectory of the moving object [17]. The curve $p(t)$ in spatio-temporal domain is the space-time path or temporal trajectory of the moving object (Fig. 1). At each time moment, a moving object exists at a position in space, but it may visit a spatial position at various time moments. The variation of an attribute is a function from a continuous domain of time to a continuous or discrete domain of the attribute, $a: t \mapsto a(t)$, or from a continuous domain of location to a continuous or discrete domain of the attribute, $a: p \mapsto a(p)$. Technically, the trajectory and attributes of a moving object may be recorded discretely with one of the methods of time-based recording, location-based recording, event-based recording, or change-based recording [18]. The time-based recording method 
applying for spatial positions of objects provides with data tuples $\left(t_{i}, p_{i}\right)$. The polyline time-ordered connecting the points of the data tuples $\left(t_{i}, p_{i}\right)$ in spatial domain approximates to the trajectory of the moving object and in spatio-temporal domain approximates to the temporal trajectory of the moving object. The time-based recording method applying for attributes $a_{m}$ provides with data tuples $\left(t_{i}, a_{m}^{i}\right)$. The location-based recording method applying for attributes $a_{n}$ provides with data tuples $\left(p_{j}, a_{n}^{j}\right)$. The variation of an attribute is inferred from the tuples $\left(t_{i}, a_{m}^{i}\right),\left(p_{j}, a_{n}^{j}\right)$ and the specification of the attribute.

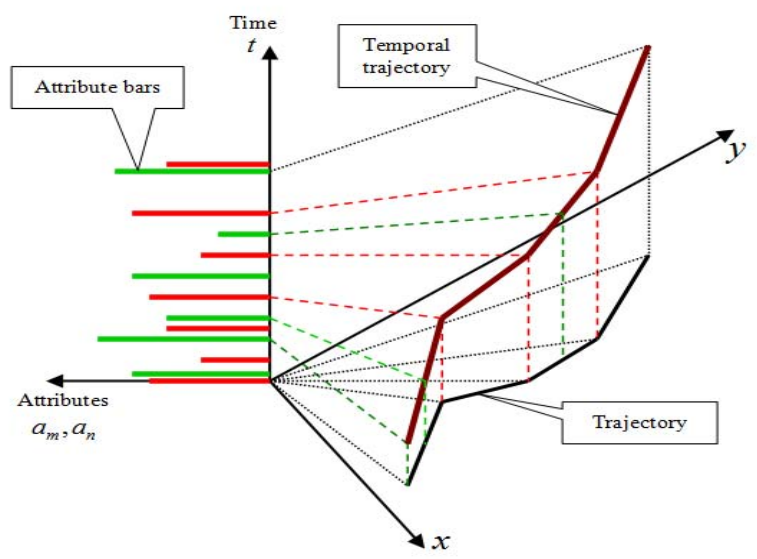

Fig. 4. The ungiven route mode of multivariate cube

3) Converting data tuples into records of a data table: The movement of an object is recorded by the data tuples $\left(t_{i}, p_{i}\right),\left(t_{i}, a_{m}^{i}\right),\left(p_{j}, a_{n}^{j}\right)$. In order to represent the movement data on a coordinate system, the data tuples as mentioned above are converted to the records of a data table of the fields $t_{i}, p_{i}, p_{j}, a_{m}, a_{n}$ as the Table I.

TABLE I: DATA TABLE FOR VISUAL REPRESENTATION

\begin{tabular}{ccccc}
\hline \hline$t_{i}$ & $p_{i}$ & $p_{j}$ & $a_{m}$ & $a_{n}$ \\
\hline $\mathrm{X}$ & $\mathrm{X}$ & & $\mathrm{X}$ & \\
\hline & & $\mathrm{X}$ & & $\mathrm{X}$ \\
\hline $\mathrm{X}$ & $\mathrm{X}$ & $\mathrm{X}$ & & $\mathrm{X}$ \\
\hline \hline
\end{tabular}

\section{Multivariate Cube FOR Representing Moving OBJECTS}

Several articles represent spatio-temporal data of the tuples $<$ time, position $>$ and spatio-attribute data of the tuples $<$ position, attribute $>$ on two separated space-time cubes because the number of dimensions of a space-time cube is less than the number of data variables [13], [7], [5]. There are three dimensions in a space-time cube; meanwhile the number of data variables includes one variable of time, one or two variables of positions, and several variables of attributes. Hence, it is difficult to represent all variables of data in a space-time cube.

In this article, we propose a cube representing the multivariable data of moving objects, called Multivariate
Cube (MC). Multivariate cubes have two display modes as follows.

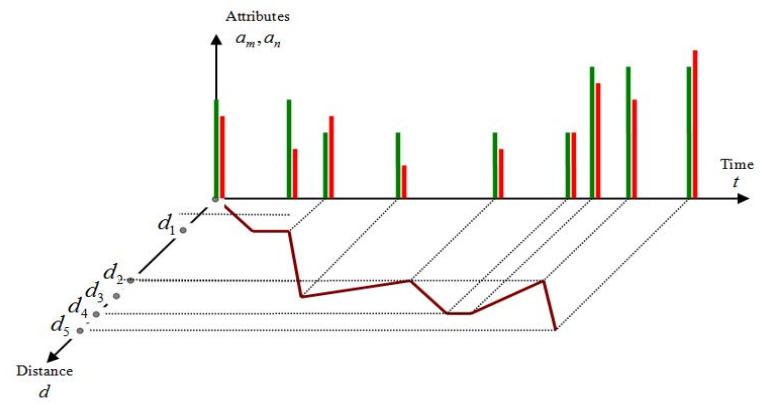

Fig. 5. The given route mode of multivariate cube

1) The mode of ungiven route (Fig. 4): This mode is applied for moving objects having ungiven routes. An ungiven route mode is a combination of two Cartesian coordinate systems, a 2-dimension coordinate system and a 3-dimension coordinate system. The 2-dimension coordinate system represents the variation of attributes over time; the 3-dimension coordinate system represents spatial positions changing over time. The two systems share a time axis. In the 3-dimension coordinate system, the data of spatial positions $p_{i}$ are indicated by a point of coordinates $\left(x_{i}, y_{i}\right)$ in the plane $(x, y)$ representing the study area, the data tuples $\left(t_{i}, p_{i}\right)$ are indicated by a point of coordinate $\left(t_{i}, x_{i}, y_{i}\right)$. The polyline time-ordered connecting the points $\left(x_{i}, y_{i}\right)$ approximates the trajectory of the moving object in space. The polyline time-ordered connecting the points $\left(t_{i}, x_{i}, y_{i}\right)$ approximates the temporal trajectory of the moving object. In the 2-dimension coordinate system, data tuples $\left(t_{i}, a_{m}^{i}\right)$ are shown as histogram bars along the time axis. Meanwhile, in order to represent data tuples $\left(p_{j}, a_{m}^{j}\right)$, the following references is created. Starting at the position $p_{j}=\left(x_{j}, y_{j}\right)$ of the data tuple $\left(p_{j}, a_{m}^{j}\right)$, users refer to a corresponding point on the temporal trajectory, then to the time axis to mark the point $t_{j}$, and finally, the attribute $a_{m}^{j}$ are shown as a bar $a_{m}$ at the time point $t_{j}$. In order to represent different attributes, colors are used as dimensions indicating various attribute variables.

2) The mode of given route (Fig. 5): This mode is applied for moving objects having given routes. In this case, the trajectory of a movement is extended to employ as a spatial dimension of a 3-dimension Cartesian coordinate system, which is called axis $d$ indicating the distance from the departure position to a certain position [18], [3]. Two other axes indicate time $t$ and attributes $a_{m}$. Colors are still used as different dimensions to indicate various attributes. Spatio-temporal positions are indicated by data tuples $\left(t_{i}, d_{i}\right)$, including time moment $t_{i}$ and distance $d_{i}$ from departure position to the spatial position corresponding $t_{i}$. The polyline time-ordered connecting the point $\left(t_{i}, d_{i}\right)$ on the plane $(t, d)$ approximates the space-time path of the movement. The 
data tuples $\left(t_{i}, a_{m}^{i}\right)$ of attributes $a_{m}$ are represented as attribute bars along the time axis. The data tuples $\left(d_{j}, a_{n}^{j}\right)$ of attributes $a_{n}$ are represented as the bars of attributes $a_{n}$ along the axis $t$ at the time positions that are referred from the points $d_{j}$ of $\left(d_{j}, a_{n}^{j}\right)$ on the axis $d$ to the space-time path, then to the time axis $t$ (Fig. $5)$.

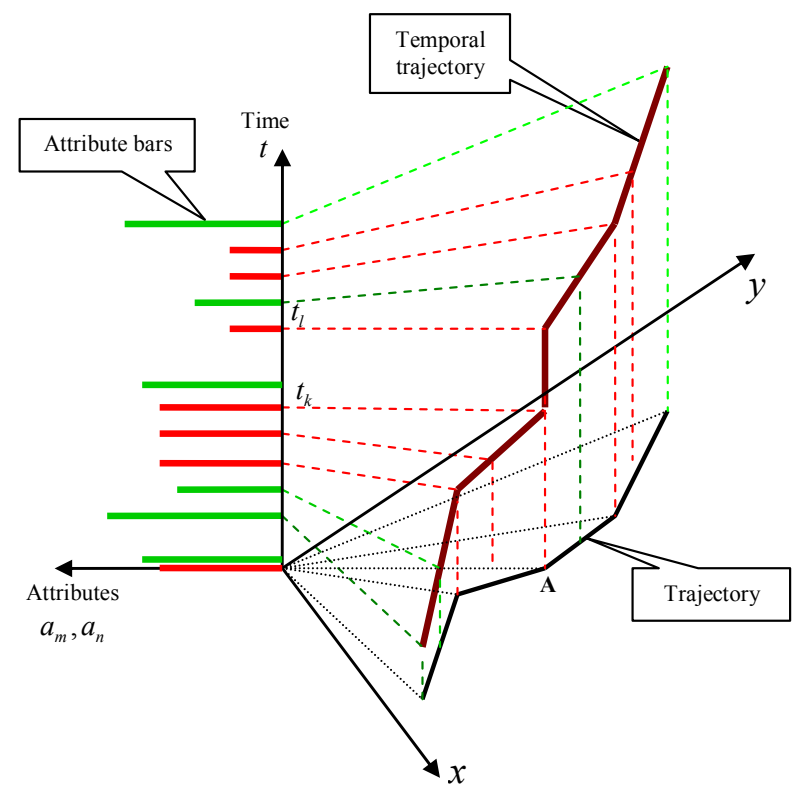

Fig. 6. The object stops at $A$ from $t_{k}$ to $t_{l}$

\section{CONCLUSION AND FUTURE WORKS}

The model of Multivariate Cube (MC) is proposed to represent multivariate data of moving objects in Cartesian coordinate systems. The multivariate cube provides with two different modes of display for ungiven route and given route. For the ungiven route mode, multivariate cube shares the time axis for representing the positions and representing the attributes of moving objects. Positions are indicated on a 2-dimension plane, and attributes as histogram bars along the time axis. For the given route mode, multivariate cube indicates data of positions on an axis representing the distance from departure place to a certain place, and attributes as histogram bars along the time axis. Accordingly, the model of multivariate cube can represent the data of positions and attributes of moving objects in a cube of Cartesian coordinate system.

The model of multivariate cube may be applied for available data or data from real-time information systems for tracking transportation means [3]. In reality, an object may stop at some place for awhile and its some attributes change, e.g. in the Fig. 6, the object stops at $A$ from $t_{k}$ to $t_{i}$. Hence, one of our future works is to implement a tool switching between two modes of multivariate cube to show the change of attributes during stop time of the object.

\section{REFERENCES}

[1] Y. Song and H. J. Miller, "Exploring traffic flow databases using space-time plots and data cubes," Transportation, vol. 39, no. 2, pp. 215-234, 2012
[2] P. Gatalsky, N. Andrienko, and G. Andrienko, "Interactive analysis of event data using space-time cube," in Proc. $8^{\text {th }}$ International Conference on Information Visualisation (IV'04) IEEE Computer Society, 2004.

[3] P. V. Tran and H. T. Nguyen, "Visualization cube for tracking moving object," in Proc. of Computer Science and Information Technology, Information and Electronics Engineering, IACSIT Press, 2011, vol. 6, pp. $258-262$.

[4] G. Andrienko and N. Andrienko, "Dynamic time transformation for interpreting clusters of trajectories with space-time cube," presented at IEEE Symposium on Visual Analytics Science and Technology, Poster, 2010.

[5] X. Li and M. J. Kraak, "New views on multivariable spatiotemporal data: the space time cube expanded," in Proc. Inter. Sym. on Spatio-temporal Modelling, Spatial Reasoning, Analysis, Data Mining and Data Fusion, XXXVI, 2005, pp. 199-201.

[6] C. Tominski, P. Schulze-Wollgast, and H. Schumann, "3D Information visualization for time dependent data on maps," in Proc. $4^{\text {th }}$ Inter. Conf. on Information Visualization, IEEE Computer Society, 2005, pp. 175-181.

[7] M. J. Kraak, "The space-time cube revisited from a geovisualization perspective," in Proc. 21st Inter. Cartographic Conf. (ICC) Cartographic Renaissance, 2003, pp. 1988-1996.

[8] T. Hagerstrand, "What about people in regional science?" presented at $9^{\text {th }}$ European Congress of Regional Science Association, vol. 24, pp. $7-21,1970$.

[9] G. Andrienko, N. Andrienko, P. Bak, D. Keim, S. Kisilevich, and S. Wrobel, "A conceptual framework and taxonomy of techniques for analyzing movement," Journal of Visual Languages and Computing, vol. 22, pp. 213-232, 2011.

[10] D. J. Peuquet, "It's About Time: A conceptual framework for the representation of temporal dynamics in geographic information systems," Annals of the Association of American Geographers, vol. 84, no. 3, pp. 441-461, September 1994.

[11] S. Dodge, R. Weibel, and A. K. Lautenschütz, "Towards a taxonomy of movement patterns," Information Visualization, vol. 2008, no. 7, pp. 240-252, 2008.

[12] M. Friendly, "Visions and re-visions of Charles Joseph Minard," Journal of Educational and Behavioral Statistics, vol. 27, no. 1, pp. $31-51,2002$.

[13] M. J. Kraak, "Geovisualization illustrated," ISPRS Journal of Photogrammetry \& Remote sensing, vol. 57, pp. 390-399, 2003.

[14] N. Andrienko, G. Andrienko, S. Hendrik, T. Liebig, and D. Hecker, "Visual analytics for understanding spatial situations from episodic movement data," Künstliche Intelligenz, vol. 26, no. 3, pp. 241-251, 2012.

[15] G. Andrienko, N. Andrienko, and M. Heurich, "An event-based conceptual model for context-aware movement analysis," International Journal Geographical Information Science, vol. 25, no. 9, pp. 1347-1370, 2011.

[16] N. Willems, R. Willem, G. Vries, J. H. M. Janssens, and V. Malais, “An integrated approach for visual analysis of a multi-source moving objects knowledge base," International Journal of Geographical Information Science, vol. 24, no. 9, pp. 1-16, 2010.

[17] G. Andrienko and N. Andrienko, "Spatio-temporal aggregation for visual analysis of movements," presented at IEEE Symposium on Visual Analytics Science and Technology, Columbus, Ohio, USA, October 21-23, 2008.

[18] N. Andrienko, G. Andrienko, N. Pelekis, and S. Spaccapietra, "Basic concepts of movement data," in Mobility, Data Mining and Privacy, Geographic Knowledge Discovery, Ed. F. Giannotti and D. Pedreschi, Springer, 2008, ch. 2, pp. 15-38.

Hong Thi Nguyen obtained Bachelor of Science in 2003, then Master in Information Technology in 2009 at University of Information Technology. She is working for the Rubber Industrial College as a lecturer and is Ph.D. student of University of Information Technology with researches on representation of multivariate data. Her contact is hongnguyen1611@gmail.com

Hung Thanh Ngo obtained doctor in 2009 in Russia, specializing on analysis, control and information processing. He is working for University of Information Technology as a lecturer and the head of information system laboratory. His interest is construction and 
optimization of information system. His contact is hungnt@uit.edu.vn

Xanh Van Nguyen obtained Master of Science in GIS in 2005. He is working for University of Information Technology as a lecturer and vice director if the office for science and technology. His interest is to develop GISystems for natural disaster management. His contact information is xanhnv@uit.edu.vn

Diem Ngoc Nguyen obtained Bachelor of Science in 2008. She is working for Axon Active Viet Nam and is a Master student of the University of Information Technology. Her interests include spatio-temporal representation, geo-visualization. Her contact information is ngngdiem@yahoo.com

Phuoc Vinh Tran is Associate Professor of Informatics, Vice Rector and Chair of the GIS Program at the University of Information Technology (UIT) in Vietnam. He received his Ph.D. in Informatics from Vietnam National University in 1994. From 1994 to 2006, he was founder and Director of the Center for Developing
IT and GIS (DITAGIS), one of the first research institutes about GIS in Vietnam in 1990s. Since 1994, he has also been founder and leader of Annual International Conference of GIS, the largest GIS event in Vietnam, significantly supported by Vietnam Ministry of Science and Technology (MOST). In 2006, he moved to University of Information Technology (UIT) and served as Vice Rector and Chair of the GIS Program. He has been PI of many GIS researches and developments since the early 1990s. He was editor of the International Journal of Geoinformatics and country editor of the GIS Development magazine. He is a senior member of IACSIT. In 2005, he obtained the gold medal from MOST for his contribution to the development of science and technology, specially GIS in Vietnam. His interests include representation of multivariate data, real-time geo-visualization, access control in government GISystems, and real-time spatial information system, real-time GIS for disaster and climate change. He has published 4 GIS books in Vietnamese and over 50 papers at international conferences and journals. His contact information is Phuoc.gis@uit.edu.vn; Phuoc.gis@gmail.com 